HNO 2022 $\cdot 70: 102-109$

https://doi.org/10.1007/s00106-021-01076-3

Angenommen: 18. Mai 2021

Online publiziert: 25. Juni 2021

๑) Der/die Autor(en) 2021

\title{
Prognosefaktoren für das Gesamtüberleben bei Oropharynxkarzinomen in Abhängigkeit vom HPV-Status
}

\begin{abstract}
Momentan steht die SARS-CoV-2(,severe acute respiratory syndrome coronavirus type $2^{\prime \prime}$ )-Pandemie weltweit im Mittelpunkt. Doch auch andere viral ausgelöste Erkrankungen verbreiten sich in unserer Bevölkerung mit schwerwiegenden Folgen. Dazu zählt die Infektion mit dem humanen Papillomavirus (HPV), die in den letzten Jahrzehnten immer mehr an Bedeutung gewonnen hat. Für die Entstehung der Plattenepithelkarzinome des Oropharynx (OSCC) spielen vor allem der High-risk-HPV-Typ 16 (HPV16) sowie Nikotin- und Alkoholabusus die maßgebliche Rolle.
\end{abstract}

Während die Inzidenzraten der KopfHals-Karzinome parallel mit der Abnahme des Tabakkonsums insgesamt rückläufig sind, wird weltweit ein Anstieg der HPV-positiven OSCC mit deutlichen geographischen Unterschieden verzeichnet [12, 24]. Aufgrund der unterschiedlichen Karzinogenese und Prognose werden die HPV-positiven und -negativen OSCC seit 2017 in der UICC 8 (Union Internationale contre le Cancer) als separate Entitäten klassifiziert. Mehrere europäische Studien berichten jedoch über eine unzureichende Prognosestratifikation der HPV-positiven OSCC zwischen einzelnen Stadien der UICC 8 [6, 19, 27]. Der Einschluss weiterer Prognosefaktoren neben HPV und TNM könnte diese optimieren.

Aktuell werden in zahlreichen klinischen Studien Strategien von spezifischen, zielgerichteten sowie deinten- sivierten Therapien bei HPV-positiven OSCC untersucht, um eine verbesserte Lebensqualität ohne Verschlechterung der Prognose zu erreichen [15]. Zwei große multizentrischen Studien $[9,16]$, die den Ersatz einer platinbasierten Chemotherapie durch eine Antikörpertherapie mit Cetuximab bei simultaner Radiotherapie verglichen, konnten jedoch keine Vorteile im Therapiearm mit Cetuximab in der Behandlung HPVinduzierter OSCC herausstellen.

Das Ziel der vorliegenden Studie war die Überprüfung ausgewählter Prognosefaktoren, wie Nikotin, Alkohol und der Therapie in Abhängigkeit des Nachweises von HPV16-spezifischer DNA (HPV16DNA) im Tumorgewebe, sowie die Analyse der Risikostratifizierung der UICC 8 bezogen auf das Gesamtüberleben (GÜ) in dem vorliegendem primär chirurgisch behandelten Patientenkollektiv.

\section{Material und Methoden}

Insgesamt wurden 524 Patienten mit der Erstdiagnose eines OSCC berücksichtigt, die in der HNO-Klinik des Universitätsklinikums Münster im Zeitraum von 2000-2016 behandelt wurden. Es lagen Genehmigungen der Ethikkommissionen der Ärztekammer Westfalen-Lippe und der Westfälischen Wilhelms-Universität (2013-511-f-S) vor. Die Daten wurden anhand der digitalen Krankenakten akquiriert und pseudonymisiert. Der Beobachtungszeitraum erstreckte sich vom Zeitpunkt der Erstdiagnose bis zum letzten Tumornachsorgetermin bzw. bis zum Versterben des Patienten.
Alkoholkonsum an mindestens $4 \mathrm{Ta}$ gen in der Woche wurde als häufiger Alkoholkonsum klassifiziert. Patienten mit einem Nikotinkonsum $\geq 10$ Packungsjahre (PY) wurden als intensive Raucher charakterisiert. Die Therapie wurde in folgenden Kategorien klassifiziert:

- alleinige Tumoroperation (OP; $n=42$ ),

- Operation und adjuvante Radiatio (OP + RT; $n=139)$,

- Operation und adjuvante Radiochemotherapie (OP + RCT; $n=146)$ und

- primäre Radiochemotherapie (RCT; $n=92$ ).

Für die Überlebensanalyse hinsichtlich der Therapie wurden die Patienten mit vollendeter primärer kurativer Therapie und von den operierten Patienten $(n=327)$ nur diejenigen mit sicheren R0-Resektionen $(n=251)$ eingeschlossen, um mögliche Verzerrungen wegen adjuvanter Therapieeskalation bei einem positiven R-Status $\mathrm{zu}$ vermeiden. Bei vorhandenen Risikofaktoren, wie nodaler Metastasierung, kapselüberschreitendem Wachstum, knappen tumorfreien oder positiven Resektionsrändern, wurde durch die postoperative Tumorkonferenz die Indikation für eine adjuvante Therapie gestellt. Standardmäßig erfolgte eine Strahlentherapie bei primärer Therapie mit einer Strahlendosis bis $72 \mathrm{~Gy}$ und im adjuvanten Setting bis $66 \mathrm{~Gy}$ über 6 Wochen. Die Chemotherapie erfolgte in erster Linie mit Cisplatin (bis $2011: 2-3 \times 100 \mathrm{mg} / \mathrm{m}^{2}$, ab 2012: $\left.5-6 \times 40 \mathrm{mg} / \mathrm{m}^{2}\right)$. 


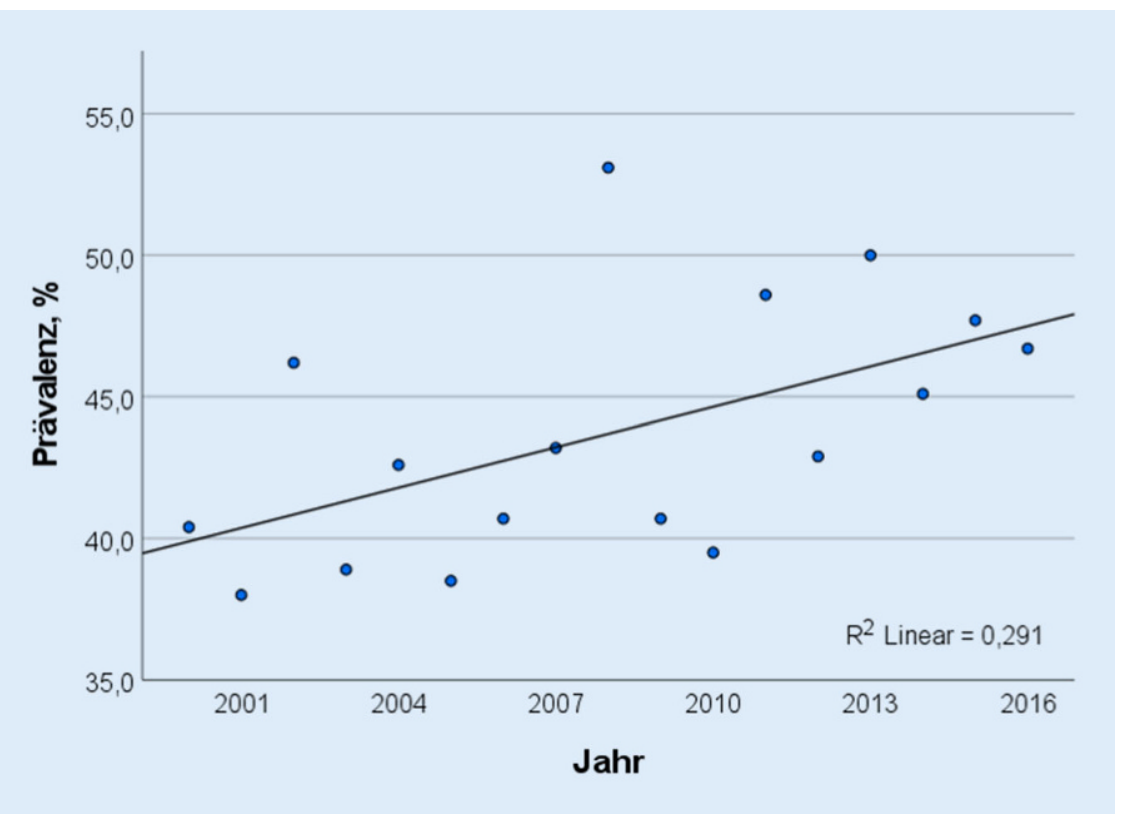

Abb. 1 ^ Prävalenzanstieg der HPV16-DNA-positiven OSCC $(n=238)$, HNO-Klinik Universitätsklinikum Münster 2000-2016. UICC Union Internationale contre le Cancer

\section{Nachweis von HPV16-DNA}

Das im Rahmen der vorliegenden Studie verwendete Gewebe entstammte einem diagnostischen Routineeingriff und wurde neben der standardisierten Histopathologie des Gerhard-Domagk-Instituts für Pathologie Münster auf HPV16DNA untersucht. Die formalinfixierten, paraffineingebetteten Gewebestücke wurden zunächst in $20 \mu \mathrm{m}$ dünne Schichten geschnitten und entparaffiniert. Im Anschluss wurde die DNA mittels eines kommerziellen Kits (QIAamp DNA Mini Kit, QIAGEN, Hilden) gemäß dem Protokoll des Herstellers isoliert. Humanes $\beta$-Globin-Gen wurde als Indikator für eine erfolgreiche Extraktion einer ausreichend großen Menge DNA angewendet. Anschließend wurden HPV16DNA der Onkogene E6 und E7 mittels Echtzeit-Polymerasekettenreaktion (ABI Prism 7900HT Sequenzerkennungssystem, TaqMan Genotyping PCR Master Mix, Applied Biosystems, Darmstadt, Deutschland) amplifiziert. Aus der ca. 600 Kopien im Genom integrierte HPV16-DNA enthaltenden humanen Zervixkarzinomzelllinie CaSki (ATCC $^{\circledR}$ CRL-1550 ${ }^{\mathrm{m}}$, Mananssas, VA, USA) isolierte DNA wurde als positive Kontrolle genutzt. Von Tonsillengewebe eines 4-jährigen Kindes nach einer Tonsillo- tomie isolierte DNA wurde als negative Kontrolle angewendet.

\section{Statistische Analyse}

Die gesammelten Daten wurden mithilfe des Programms SPSS (IBM SPSS 26.0) analysiert. Zur Überprüfung der statistischen Unterschiede bezüglich der Häufigkeitsverteilung der HPV16-DNApositiven und -negativen Gruppen wurde der Pearson $\chi^{2}$-Test angewandt, zum Vergleich des medianen Lebensalters der Mann-Whitney-U-Test. Um die Prävalenzveränderungen im beobachteten Zeitraum zu überprüfen, erfolgte eine lineare Regressionsanalyse. Für die Überlebensanalyse wurden die KaplanMeier-Methode und der Log-Rank-Test angewendet. Als Signifikanzniveau wurde $p \leq 0,05$ festgelegt.

\section{Ergebnisse}

\section{Patienten- und Tumor- charakteristika}

Im vorliegenden Gesamtkollektiv betrug der Anteil der HPV16-DNA-positiven OSCC $45,4 \%(n=238 / 524)$. Außerdem wurde ein signifikanter Anstieg der jährlichen Prävalenz der HPV16-DNApositiven OSCC von $40 \%(n=12 / 30)$ im
Jahr 2000 auf $46 \%(n=18 / 39)$ im Jahr 2016 verzeichnet $(p=0,025 ; \beta=0,539$; - Abb. 1). Hinsichtlich des medianen Lebensalters der Patienten zum Zeitpunkt der Diagnosestellung zeigte sich kein statistischer Unterschied zwischen beiden Gruppen $(60,7 \pm 9,8$ vs. $60,3 \pm 10,2$ Jahre, $p=0,161$; - Tab. 1). Die HPV16DNA-positiven OSCC wiesen bei Erstdiagnose häufiger einen kleineren Primärtumor (T1-T2: 68,9\% [ $n=164]$ vs. $54,1 \% \quad[n=155], p=0,001)$ und eine lokale Metastasierung (N1-N3: 81,2\% [ $n=195]$ vs. $68,5 \%$ [ $n=196], p=0,001$; - Tab. 1) im Vergleich zu HPV16-DNAnegativen OSCC auf. Das Auftreten von Fernmetastasen bei Erstdiagnose war nicht vom HPV16-DNA-Status abhängig (M1: $4,6 \%(n=11)$ vs. $7,0 \%$ $(n=20), p=0,337$; \ab. 1). Die Patienten mit einem HPV16-DNA-positiven OSCC wiesen signifikant seltener einen kumulativen Nikotinkonsum $\geq 10 \mathrm{PY}$ $(63,0 \%[n=150]$ vs. $84,6 \%[n=242]$, $p<0,001)$ und häufigen Alkoholkon$\operatorname{sum}(31,1 \%[n=74]$ vs. $56,6 \%[n=162]$, $p<0,001)$ auf. In der HPV16-DNA-positiven Gruppe wurde signifikant häufiger eine adjuvante RCT verabreicht $(41,2 \%$ [ $n=82]$ vs. $29,1 \%[n=64], p=0,006$; - Tab. 1). Die Patienten, die einer primären RCT zugeführt wurden, zeigten häufiger ein höheres UICC-Stadium im Vergleich $\mathrm{zu}$ operierten Patienten sowohl in der HPV16-DNA-negativen (UICC-8-Stadien III-IV: 93,2\% [ $n=55]$ vs. $67,1 \%[n=108], p=0,001)$ als auch in der HPV16-DNA-positiven Gruppe (UICC-8-Stadien III-IV: 45,5\% [ $n=15]$ vs. $7,2 \%[n=12], p=0,001$; • Tab. 1$)$.

\section{Univariate Gesamtüberlebens- analyse}

Patienten mit einem HPV16-DNA-positiven OSCC zeigten eine um 18,5\% bessere 5-Jahres-Gesamtüberlebensrate (5-JGÜ) als die Patienten mit einem HPV16-DNA-negativen OSCC (88,3 vs. $69,8 \%, p<0,001$; • Tab. 2). Im Gesamtkollektiv zeigten häufiger Alkoholkonsum $(p=0,004)$ und Nikotinkonsum $\geq 10 \mathrm{PY} \quad(p=0,009$; - Tab. 2$)$ einen signifikanten negativen Einfluss auf das GÜ. Wurde der Einfluss auf das GÜ in Abhängigkeit des HPV16-DNA-Sta- 
HNO 2022 · 70:102-109 https://doi.org/10.1007/s00106-021-01076-3

(c) Der/die Autor(en) 2021

A. Riders $\cdot$ M. Oberste $\cdot$ B. Abbaspour $\cdot$ A. Beule $\cdot$ C. Rudack

Prognosefaktoren für das Gesamtüberleben bei Oropharynxkarzinomen in Abhängigkeit vom HPVStatus

\section{Zusammenfassung}

Hintergrund. Aufgrund der unterschiedlichen Karzinogenese, Prognose und klinischen Manifestation werden seit der 8. Auflage des TNM-Klassifikationssystems der UICC/AJCC (UICC 8) humane Papillomavirus (HPV)assoziierte und HPV-negative oropharyngeale Plattenepithelkarzinome (OSCC) als 2 Entitäten separat klassifiziert.

Material und Methoden. Insgesamt 524 Patienten mit einem im Zeitraum von 2000-2016 in der HNO-Klinik des Universitätsklinikums Münster behandelten OSCC wurden hinsichtlich des Nachweises von HPV-Typ-16-spezifischer DNA (HPV16-DNA), des Nikotin- und Alkoholkonsums sowie des Therapieeinflusses auf das Gesamtüberleben (GÜ) untersucht.
Ergebnisse. Ein signifikanter Anstieg der jährlichen Prävalenz der HPV16-DNApositiven OSCC von $40 \%(n=12 / 30)$ im Jahr 2000 auf 46\% $(n=18 / 39)$ im Jahr 2016 wurde verzeichnet $(p=0,025, \beta=0,539)$. $89 \%(n=212)$ der HPV16-DNA-positiven OSCC wurden anhand der UICC 8 gegenüber der UICC 7 herabgestuft. Im Gesamtkollektiv zeigten der häufige Alkohol- und Nikotinkonsum ( $\geq 10$ Packungsjahre) einen statistisch relevanten negativen Einfluss auf das GÜ ( $p=0,004$ und $p=0,009)$. Auch häufiger Alkoholkonsum war in der HPV16DNA-negativen Gruppe prognoserelevant $(p=0,049)$. In der HPV16-DNA-positiven Gruppe zeigte sich bezüglich des GÜ zwischen den UICC-Stadien I und II $(p=0,481)$ sowie zwischen III und IV $(p=0,439)$ gemäß UICC 8 kein statistischer Unterschied.

Schlussfolgerungen. Die UICC 8 verbessert zwar die Prognosestratifikation der OSCC durch die Trennung von HPV-positiven und HPV-negativen Tumoren im Vergleich zu UICC 7, die prognostische Aussagekraft der UICC 8 für die HPV-assoziierten OSCC ist jedoch weiterhin unzureichend. Der Noxenkonsum könnte zukünftig Einfluss auf die UICC-Klassifikation nehmen, um die prognostische Aussagekraft weiter zu verbessern.

Schlüsselwörter

Humanes Papillomavirus - Oropharynxkarzinom · Alkoholkonsum · Nikotinkonsum . Gesamtüberleben

\section{Prognostic factors for overall survival in oropharyngeal carcinoma depending on HPV status}

\section{Abstract}

Background. Due to their differing carcinogenesis, prognosis and clinical manifestation, human papillomavirus (HPV)-associated and HPV-negative oropharyngeal squamous cell carcinoma (OSCC) have been classified separately as two entities since the 8 th edition of the AJCC/UICC TNM staging system (UICC 8). Materials and methods. A total of 524 patients with OSCC treated between 2000 and 2016 at the Department of Otorhinolaryngology, Head and Neck Surgery of the University Hospital Muenster, Germany, were examined for the detection of HPV type 16-specific DNA (HPV16-DNA), nicotine and alcohol consumption and the influence of therapy on overall survival (OS).
Results. There was a significant increase in the annual prevalence of HPV16-DNA-positive OSCC from $40 \%(n=12 / 30)$ in 2000 to $46 \%$ $(n=18 / 39)$ in $2016(p=0.025, \beta=0.539)$. Of the HPV16-DNA-positive OSCC, $89 \%(n=212)$ were downgraded on the basis of UICC 8 compared to UICC 7. In the overall collective, frequent alcohol and nicotine ( $\geq 10$ pack years) consumption showed a significant negative influence on OS ( $p=0.004$ and $p=0.009$, respectively). Frequent alcohol consumption was also prognostically relevant in the HPV16DNA-negative group $(p=0.049)$. In the HPV16DNA-positive group, the prognosis for OS according to UICC 8 showed no statistically significant difference between stages I and II $(p=0,481)$, or between III and IV $(p=0.439)$. Conclusions. The current UICC 8 improves the prognostic stratification of OSCC due to the separation of HPV-positive and HPV-negative tumors in comparison to UICC 7. However, the prognostic significance of UICC 8 for HPVassociated OSCC is still insufficient. In the future, alcohol and nicotine consumption could influence the UICC classification in order to further improve prognostic significance.

\section{Keywords}

Human papillomavirus · Oropharyngeal cancer - Nicotine consumption - Alcohol consumption · Overall survival tus vergleichend betrachtet, ergab sich jedoch ein statistisch negativer Einfluss von häufigem Alkoholkonsum auf das GÜ nur bei Patienten mit HPV16DNA-negativem OSCC $(p=0,049)$. Die Patienten mit Nikotinkonsum $\geq 10 \mathrm{PY}$ wiesen ein schlechteres GÜ sowohl in der HPV16-DNA-negativen als auch in der HPV16-DNA-positiven Gruppe auf, aber es ergab sich keine statistische Signifikanz $(p=0,322$ und $p=0,095$; - Tab. 2). Innerhalb der HPV16-DNA- negativen Gruppe wiesen die Patienten, die eine primäre RCT (5-JGÜ 56,3\%) erhalten hatten, ein signifikant schlechteres GÜ im Vergleich zu Patienten nach OP, OP + RT und OP + RCT auf (5-JGÜ $91,5 \%, p=0,005 ; 75,9 \%, p=0,005$ und 90,7\%, $p=0,003$; - Tab. 2; • Abb. 2). Innerhalb der HPV16-DNA-positiven Gruppe zeigten die Patienten nach primärer RCT (5-JGÜ 86,3\%) wiederum keinen signifikanten Unterschied des GÜ im Vergleich zu Patienten nach OP,
$\mathrm{OP}+\mathrm{RT}$ und OP + RCT (5-JGÜ 87,8\%, $p=0,549 ; 88,6 \%, p=0,728$ und $98,0 \%$, $p=0,112$; ・ Tab. 2; • Abb. 2).

\section{Stratifikation gemäß UICC 7 und UICC 8}

Neunundachtzig Prozent $(n=212)$ der HPV16-DNA-positiven OSCC wurden anhand der UICC 8 gegenüber der UICC 7 einem niedrigeren Stadium zugeordnet. Gemäß UICC 7 befand sich 


\begin{tabular}{|c|c|c|c|c|c|c|c|}
\hline \multicolumn{2}{|l|}{ Parameter } & \multicolumn{3}{|c|}{$\begin{array}{l}\text { HPV16-DNA-negativ } \\
n=286(54,6 \%)\end{array}$} & \multicolumn{2}{|c|}{$\begin{array}{l}\text { HPV16-DNA-positiv } \\
n=238(45,4 \%)\end{array}$} & $p$-Wert \\
\hline \multicolumn{2}{|l|}{ Alter (Jahre) } & \multicolumn{3}{|l|}{$60,7 \pm 9,8$} & \multicolumn{2}{|l|}{$60,3 \pm 10,2$} & $p=0,161$ \\
\hline \multicolumn{2}{|l|}{-} & \multicolumn{3}{|l|}{$n(\%)$} & \multicolumn{2}{|l|}{$n(\%)$} & - \\
\hline \multirow[t]{2}{*}{ Geschlecht } & Weiblich & \multicolumn{3}{|l|}{$64(22,5)$} & \multicolumn{2}{|l|}{$66(27,6)$} & \multirow[t]{2}{*}{$p=0,187$} \\
\hline & Männlich & \multicolumn{3}{|l|}{$222(77,6)$} & \multicolumn{2}{|l|}{$172(72,2)$} & \\
\hline \multirow[t]{2}{*}{ T-Status } & $T 1-T 2$ & \multicolumn{3}{|l|}{$155(54,1)$} & \multicolumn{2}{|l|}{$164(68,9)$} & \multirow[t]{2}{*}{$p=0,001$} \\
\hline & T3-T4 & \multicolumn{3}{|l|}{$131(45,8)$} & \multicolumn{2}{|l|}{$74(31,0)$} & \\
\hline \multirow[t]{2}{*}{$N$-Status } & NO & \multicolumn{3}{|l|}{$90(31,5)$} & \multicolumn{2}{|l|}{$43(18,0)$} & $p=0,001$ \\
\hline & $N 1-N 3$ & \multicolumn{3}{|l|}{$196(68,5)$} & \multicolumn{2}{|l|}{$195(81,2)$} & \\
\hline \multirow[t]{2}{*}{ M-Status } & MO & \multicolumn{3}{|l|}{$266(93,0)$} & \multicolumn{2}{|l|}{$227(95,4)$} & $p=0,337$ \\
\hline & M1 & \multicolumn{3}{|l|}{$20(7,0)$} & \multicolumn{2}{|l|}{$11(4,6)$} & \\
\hline \multirow[t]{2}{*}{ Nikotinkonsum } & $<10 P Y$ & \multicolumn{3}{|l|}{$44(15,3)$} & $88(36,9)$ & & $p<0,001$ \\
\hline & $\geq 10 P Y$ & $242(84,6)$ & & & $150(63,0)$ & & \\
\hline Alkoholkonsum & Kein/gelegentlich & $124(43,4)$ & & & $164(68,9)$ & & $p<0,001$ \\
\hline & Häufig & $162(56,6)$ & & & $74(31,1)$ & & \\
\hline Therapie & Alle & $O P$ & $25(11,4)$ & & $17(8,5)$ & & $p=0,017$ \\
\hline & & $O P+R T$ & $72(32,7)$ & & $67(33,7)$ & & \\
\hline & & $O P+R C T$ & $64(29,1)$ & & $82(41,2)$ & & \\
\hline & & Primäre $R C T$ & $59(26,8)$ & & $33(16,6)$ & & \\
\hline & $O P+R C T$ vs. andere & $O P+R C T$ & $64(29,1)$ & & $82(41,2)$ & & $p=0,006$ \\
\hline & Therapie & Andere Therapie & $156(70,1)$ & & $117(58,8)$ & & \\
\hline- & & HPV16-DNA-neg & & & HPV16-DN & & \\
\hline & & $\begin{array}{l}O P \pm R(C) T \\
n(\%)\end{array}$ & $\begin{array}{l}\text { Primäre RCT } \\
n(\%)\end{array}$ & $p$-Wert & $\begin{array}{l}O P \pm R(C) T \\
n(\%)\end{array}$ & $\begin{array}{l}\text { Primäre RCT } \\
n(\%)\end{array}$ & $p$-Wert \\
\hline UICC 8 & $I-I I$ & $53(32,9)$ & $4(6,6)$ & $p<0,001$ & $154(92,8)$ & $18(54,5)$ & $p<0,001$ \\
\hline & $I I I-I V$ & $108(67,1)$ & $55(93,2)$ & & $12(7,2)$ & $15(45,5)$ & \\
\hline
\end{tabular}

\section{Tab. 2 Univariate Analyse des Gesamtüberlebens}

\begin{tabular}{|c|c|c|c|c|c|c|c|c|c|c|}
\hline \multicolumn{2}{|l|}{ Parameter } & \multicolumn{3}{|c|}{ Gesamtkollektiv } & \multicolumn{3}{|c|}{ HPV16-DNA-negativ } & \multicolumn{3}{|c|}{ HPV16-DNA-positiv } \\
\hline \multirow{2}{*}{$\begin{array}{l}\text { HPV16-DNA- } \\
\text { Status }\end{array}$} & Negativ & $\begin{array}{l}\boldsymbol{n}(\%) \\
286(54,6)\end{array}$ & $\begin{array}{l}\text { 5-JGÜ } \\
\text { (\%) }\end{array}$ & $\begin{array}{l}\boldsymbol{p} \text {-Wert } \\
p<0,001\end{array}$ & $\begin{array}{l}n(\%) \\
-\end{array}$ & 5-JGÜ, (\%) & $p$-Wert & $n(\%)$ & $\begin{array}{l}\text { 5-JGÜ } \\
(\%)\end{array}$ & \\
\hline & Positiv & $238(45,4)$ & 88,3 & & & & & & & \\
\hline \multirow[t]{2}{*}{ Geschlecht } & Weiblich & $130(24,8)$ & 87,1 & $p=0,073$ & $64(22,5)$ & 80,4 & $p=0,087$ & $66(27,6)$ & 90,9 & $p=0,775$ \\
\hline & Männlich & $394(75,2)$ & 76,4 & & $222(77,6)$ & 66,9 & & $172(72,2)$ & 86,9 & \\
\hline \multirow{2}{*}{$\begin{array}{l}\text { Nikotinkonsum } \\
\text { (PY) }\end{array}$} & $<10$ & $132(25,2)$ & 87,2 & $p=0,009$ & $44(15,3)$ & 78,9 & $p=0,322$ & $88(36,9)$ & 90,7 & $p=0,095$ \\
\hline & $\geq 10$ & $392(74,8)$ & 75,7 & & $242(84,6)$ & 68,3 & & $150(63,0)$ & 85,9 & \\
\hline \multirow[t]{2}{*}{ Alkoholkonsum } & $\begin{array}{l}\text { Kein/ } \\
\text { gelegentlich }\end{array}$ & $288(55,0)$ & 84,2 & $p=0,004$ & $124(43,4)$ & 79,1 & $p=0,049$ & $164(68,9)$ & 87,7 & $p=0,539$ \\
\hline & Häufig & $236(45,0)$ & 70,6 & & $162(56,6)$ & 63,0 & & $74(31,1)$ & 86,2 & \\
\hline \multirow[t]{4}{*}{ Therapie } & $\mathrm{OP}$ & $41(12,1)$ & 90,0 & $p=0,001$ & $24(13,2)$ & 91,5 & $p=0,001$ & $17(10,8)$ & 87,8 & $p=0,160$ \\
\hline & $\mathrm{OP}+\mathrm{RT}$ & $116(34,2)$ & 82,3 & & $62(34,1)$ & 75,9 & & $54(34,4)$ & 88,6 & \\
\hline & $\mathrm{OP}+\mathrm{RCT}$ & $94(27,7)$ & 95,2 & & $40(22,0)$ & 90,7 & & $54(34,4)$ & 98,0 & \\
\hline & Primäre RCT & $89(26,1)$ & 68,9 & & $57(31,1)$ & 56,3 & & $32(20,4)$ & 86,3 & \\
\hline
\end{tabular}




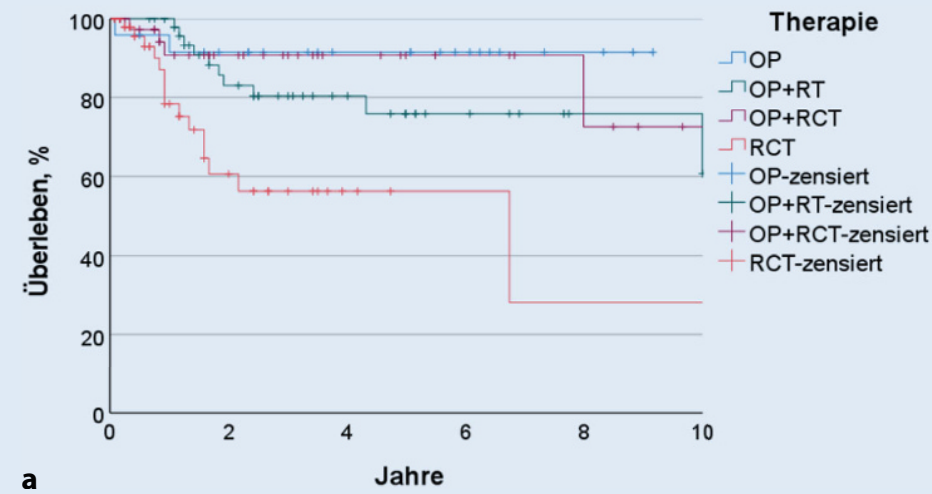

\begin{tabular}{|c|c|c|c|}
\hline Therapie & $\begin{array}{c}\mathrm{OP}, \\
\mathrm{n}=24\end{array}$ & $\begin{array}{c}\mathrm{OP}+\mathrm{RT}, \\
\mathrm{n}=62\end{array}$ & $\begin{array}{c}\mathrm{OP}+\mathrm{RCT}, \\
\mathrm{n}=40\end{array}$ \\
\hline $\begin{array}{c}\mathrm{RCT}, \\
\mathrm{n}=57\end{array}$ & $\mathbf{p}=\mathbf{0 , 0 0 5}$ & $\mathbf{p}=\mathbf{0 , 0 0 5}$ & $\mathbf{p}=\mathbf{0 , 0 0 3}$ \\
\hline
\end{tabular}
Jahre

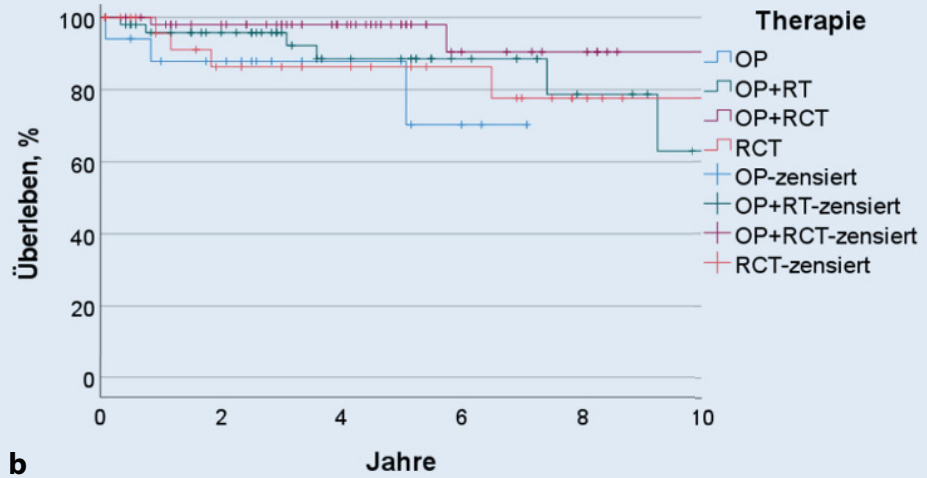

\begin{tabular}{c|c|c|c|}
\hline Therapie & $\begin{array}{c}\mathrm{OP}_{1} \\
\mathrm{n}=17\end{array}$ & $\begin{array}{c}\mathrm{OP}+\mathrm{RT}, \\
\mathrm{n}=54\end{array}$ & $\begin{array}{c}\mathrm{OP}+\mathrm{RCT}, \\
\mathrm{n}=54\end{array}$ \\
\hline $\begin{array}{c}\mathrm{RCT}, \\
\mathrm{n}=32\end{array}$ & $\mathrm{p}=0,549$ & $\mathrm{p}=0,728$ & $\mathrm{p}=0,112$ \\
\hline
\end{tabular}

Abb. $2 \Delta$ Gesamtüberleben in Abhängigkeit von Therapie und tabellarischer Überblick der Signifikanzwerte im Long-RankTest von den paarweisen Vergleichen (RCTvs. OP/OP + RT/OP + RCT) in der HPV16-DNA-negativen (a), in der HPV16-DNApositiven (b) Gruppe. OP Operation, RT Radiotherapie, RCT Radiochemotherapie, UICC Union Internationale contre le Cancer, 5-JGÜ 5-Jahres-Gesamtüberleben

der Großteil der HPV16-DNA-positiven OSCC in Stadien IV $(59,7 \%, n=142)$ und III (27,3\%, n= 65; • Abb. 3). Gemäß UICC 8 befanden sich demgegenüber wiederum mehr HPV16-DNA-positive OSCC in den Stadien I (44,5\%, $n=106)$ und II $(39,1 \%, n=93)$. In der HPV16-DNA-positiven Gruppe zeigte sich gemäß UICC 7 ein signifikant unterschiedliches GÜ lediglich zwischen den Stadien II und III $(p=0,050)$ sowie III und IV ( $p=0,035 ; \bullet$ Abb. 4). Außerdem wiesen die Patienten mit dem UICC-7Stadium III paradoxerweise ein signifikant günstigeres GÜ als die Patienten mit Stadium II (5-JGÜ 94,7 vs. 72,7\%, $p=0,050$; • Abb. 4) auf. Nach der ReKlassifizierung gemäß UICC 8 ergab sich eine signifikante Prognosestratifikation zwischen den Stadienpaaren I und III, I und IV, II und III, sowie II und IV $(p=0,001 ; p=0,001 ; p=0,017 ; p=0,002$; - Abb. 4). Es bestand jedoch weiterhin kein signifikanter Unterschied des GÜ zwischen den UICC-8-Stadien I und II $(p=0,481)$ sowie zwischen III und IV $(p=0,439)$.

\section{Diskussion}

\section{Besseres Gesamtüberleben und steigende Prävalenz}

Eine günstigere Prognose der Patienten mit einem HPV16-DNA-positiven OSCC trotz der erhöhten lokalen Metastasierungsrate steht im Einklang mit der Literatur [1, 6, 25]. Der Anstieg der jährlichen Prävalenz der HPV16-DNApositiven OSCC zwischen 2000-2016 bestätigt ebenfalls den allgemeinen Konsens über die zunehmende Verbreitung der HPV-positiven OSCC in den Industrieländern [3, 14, 24]. Die Gründe dafür sind jedoch noch nicht vollständig geklärt [25]. Obwohl in mehreren Stu- dien ein verändertes Sexualverhalten als mögliche Ursache hierfür diskutiert wird [3-5], konnte eine aktuelle Studie aus Deutschland keine Korrelation zwischen sexuellen Präferenzen und der HPVInfektion zeigen [20].

\section{Regionale Altersunterschiede}

Die vorliegenden Daten zeigten keinen statistisch signifikanten Altersunterschied zwischen Patienten mit einem HPV16-DNA-positiven und -negativen OSCC. Mehrere Publikationen aus Deutschland beschreiben die Patienten mit einem HPV-assoziierten OSCC ebenfalls als gleichaltrig mit $[1,27]$ oder sogar älter als [23] Patienten mit einem HPV-negativen OSCC. Zahlreiche USamerikanischen Studien weisen wiederum auf ein jüngeres Alter bei Patienten mit einem HPV-positiven OSCC hin [2, 22]. 


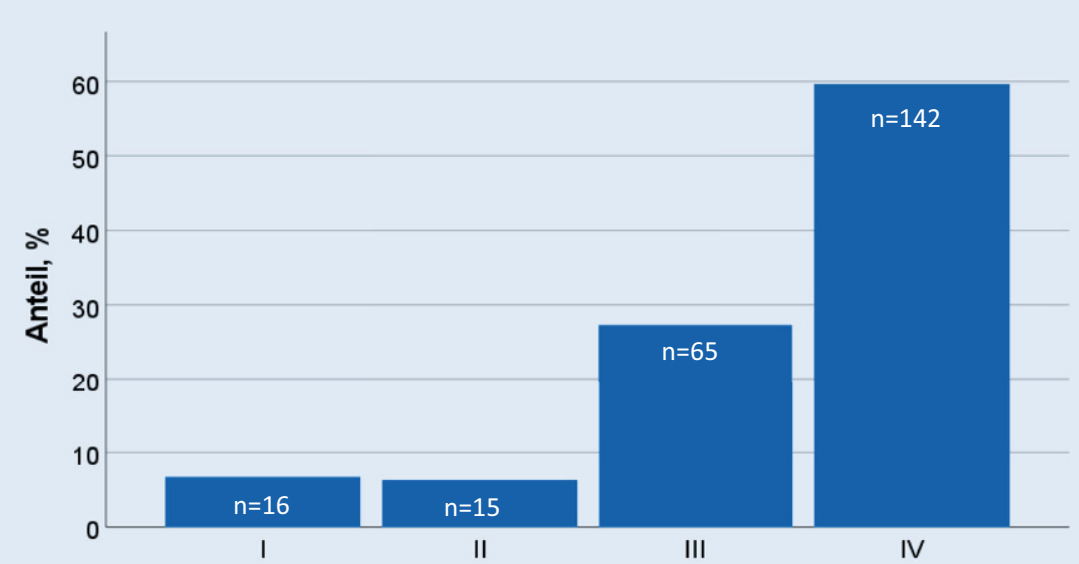

a

Stadien gemäß UICC 7

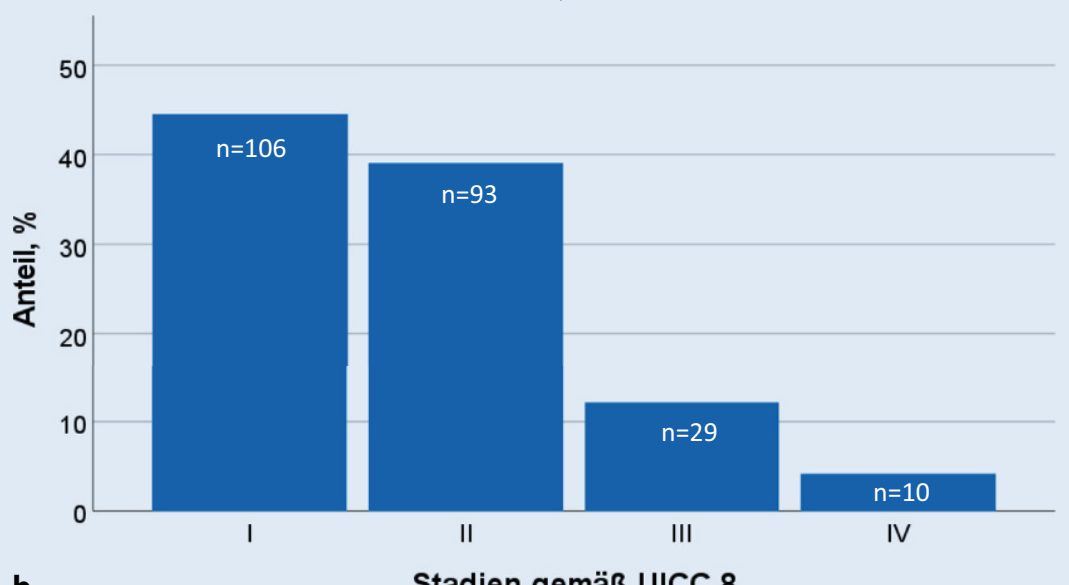

b

Stadien gemäß UICC 8

Abb. $3 \Delta$ Prozentualer Anteil der Patienten in einzelnen Stadien gemäß UICC7 (a) und UICC 8 (a) innerhalb der HPV16-DNA-positiven Gruppe

\section{Nikotin- und Alkoholabusus}

Analog zu einer vergleichbaren Studie aus Deutschland [27] ergab sich der Alkoholkonsum als ein signifikanter Prognosefaktor innerhalb des Gesamtkollektivs und in der HPV-negativen Gruppe. In einer anderen Publikation zeigten Würdemann et al. einen statistisch negativen Einfluss von häufigem Alkoholkonsum auf das GÜ bei Patienten mit einem HPVpositiven wie mit einem HPV-negativen OSCC [26]. Sowohl in der vorliegenden Untersuchung als auch in einer Publikation aus Deutschland [27] zeigte ein Tabakkonsum $\geq 10 \mathrm{PY}$ eine statistisch signifikante Assoziation zu schlechterem GÜ den, auch nach der Erstdiagnose den Nikotinkonsum zumindest zu reduzieren, da eine aktuelle Studie aus Deutschland zeigte, dass die Reduktion oder die komplette Einstellung des Tabakkonsums die Prognose verbessert [8].

\section{Prognosestratifikation gemäß UICC 8 noch nicht optimal}

In dem hier vorgestellten Patientenkollektiv spiegelt die UICC 8 die Prognose der Patienten mit HPV-assoziiertem OSCC im Vergleich zu UICC 7 besser wider. Es besteht jedoch weiterhin kein statistischer Unterschied der Prognose zwischen den UICC-8-Stadien I und II sowie III und IV. Da die absolute Mehrheit der Patienten mit HPV-assoziiertem OSCC zu den UICC-8-Stadien I und II gehören, erscheint die prognostische Aussagekraft der aktualisierten TNM-Klassifikation weiterhin unzureichend. Vergleichbare europäische Studien zeigten hinsichtlich der HPVpositiven OSCC keinen statistischen Unterschied der Prognosestratifikation zwischen den UICC-8-Stadien II und III $[6,27]$ sowie I und II [19]. Daher scheinen weitere Anpassungen erforderlich, um die Vorhersage der Überlebensprognose zu verbessern. Die Hinzunahme weiterer Prognosefaktoren in die TNMKlassifikation könnte möglicherweise die Risikostratifizierung optimieren.

\section{Therapiebezogene Unterschiede in Abhängigkeit vom HPV-Status}

Konkordant zur Literatur [1] ergab auch die vorliegende Untersuchung, dass die Patienten mit einem HPV16-DNA-positiven OSCC nach Operation statistisch häufiger eine adjuvante RCT erhielten. Dieses Phänomen ist einerseits erklärbar durch die erhöhte Neigung zu einer lokalen Metastasierung der HPV-positiven OSCC, weshalb die HPV-positiven OSCC anhand der TNM 7 einem höheren N-Status zugeordnet wurden. Andererseits gibt es Hinweise, dass der oft durch den reduzierten Noxenkonsum bedingte bessere Allgemeinzustand mit weniger Begleiterkrankungen der Patienten mit einem HPV-positiven OSCC eine adjuvante RCT häufiger ermöglicht 

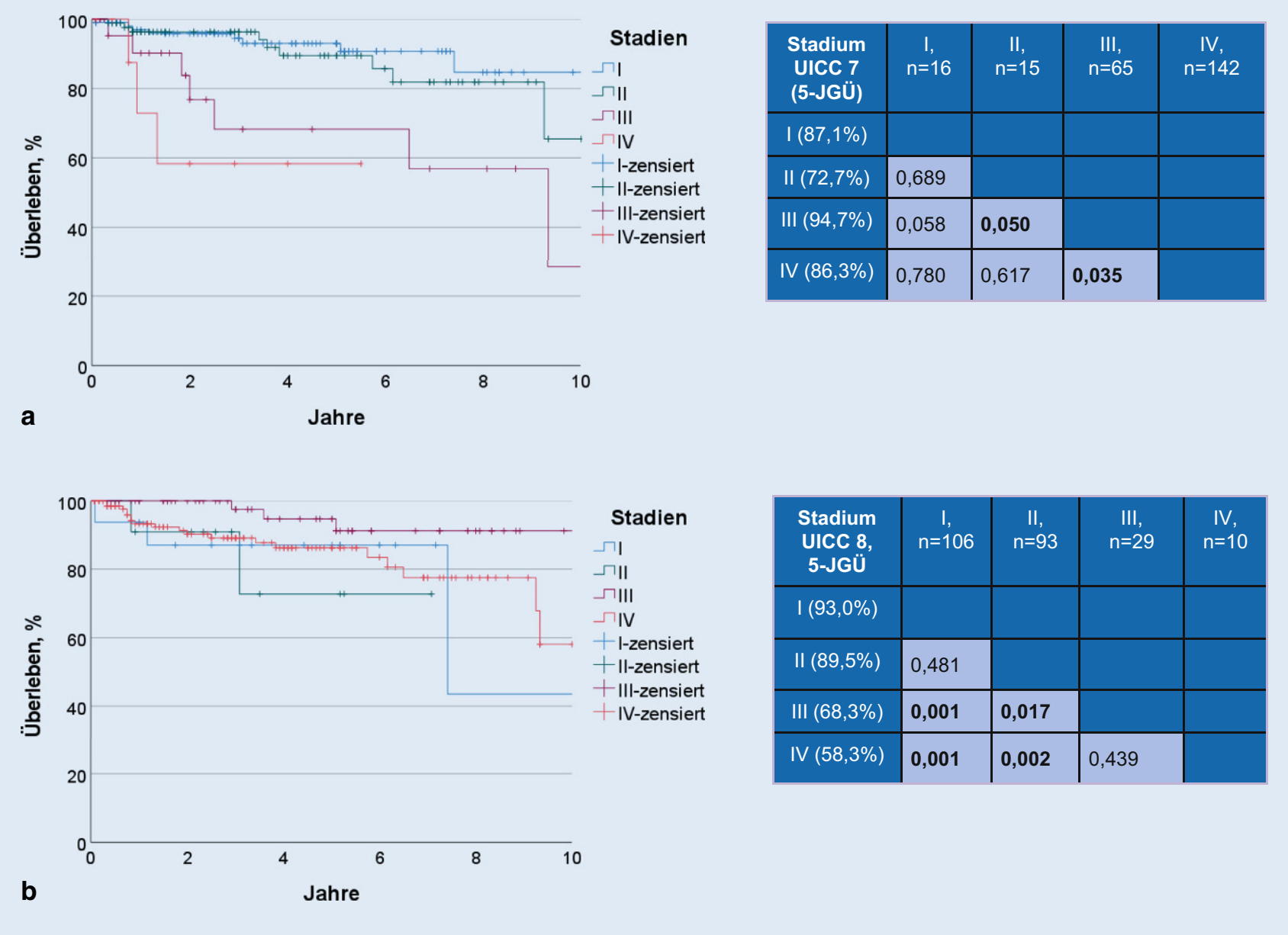

Abb. 4 \ Stratifikation des Gesamtüberlebens des HPV16-DNA-positiven Kollektivs in Abhängigkeit von UICC7 (a) und UICC 8 (b) und tabellarischer Überblick der Signifikanzwerte im Long-Rank-Test der paarweisen Vergleiche zwischen den einzelnen Stadien. 5-JGÜ 5-Jahres-Gesamtüberlebensrate

bei sonst analoger Risikokonstellation im Vergleich zu Patienten mit einem HPVnegativen OSCC [1]. In einer anderen Studie aus Deutschland zeigten Nikotinabusus und eine höhere Anzahl an Komorbiditäten wiederum keinen negativen Einfluss auf die Dosiserreichung von $\mathrm{R}(\mathrm{C}) \mathrm{T}$ (Radiatio \pm Chemotherapie) bei Patienten mit Kopf-Hals-Karzinomen [7].

Passend zur klinischen Erfahrung, dass in Deutschland typischerweise bei operablem OSCC die chirurgische Therapie bevorzugt wird, zeigten sowohl $\mathrm{Pa}$ tienten mit einem HPV16-DNA-negativen als auch Patienten mit HPV16DNApositiven OSCC, die einer primären RCT zugeführt wurden, einen größeren Anteil an höheren UICC-Stadien. Während innerhalb der HPV16-DNAnegativen Gruppe die Patienten nach primärer RCT ein schlechteres GÜ im Vergleich $\mathrm{zu}$ operierten Patienten $\mathrm{OP} \pm \mathrm{R}(\mathrm{C}) \mathrm{T}$ - zeigten, wiesen die Patienten mit einem HPV16-DNA-positiven OSCC nach primärer RCT wiederum eine mit der von operierten Patienten vergleichbare Prognose auf. Auch eine ähnliche retrospektive Studie aus Frankreich liefert Hinweise, dass die HPVassoziierten OSCC besser auf eine RCT ansprechen als HPV-negative OSCC. Während im Gesamtkollektiv die Patienten mit OSCC nach primärer RCT ein schlechteres krankheitsfreies Überleben im Vergleich zu Patienten nach $\mathrm{OP} \pm \mathrm{R}(\mathrm{C}) \mathrm{T}$ zeigten, wiesen die Patienten mit einem p16-positiven OSCC eine vergleichbare Prognose nach $\mathrm{OP} \pm \mathrm{R}(\mathrm{C}) \mathrm{T}$ und primärer RCT auf [17]. Obwohl man in der Literatur widersprüchliche Ergebnisse findet, zeigt die Mehrheit der Studien eine höhere Sensitivität der HPV-positiven Tumorzellen auf eine Radiatio sowohl in vivo [13] als auch unter In-vitro-Bedingungen [21].

\section{Fazit für die Praxis}

- In Deutschland und anderen Industrieländern wird ein Prävalenzanstieg der HPV-positiven OSCC verzeichnet.

- Die aktualisierte UICC 8 verbessert zwar die Prognosestratifikation der Patienten mit einem HPV-positiven OSCC im Vergleich zu UICC 7, ist jedoch noch nicht optimal.

- Erhöhter Noxenkonsum und assoziierte Komorbiditäten sind prognoserelevant für Patienten mit OSCC, daher könnten diese Faktoren zukünftig Einfluss auf die UICCKlassifikation nehmen. 


\section{- Obwohl es Hinweise auf eine erhöhte Strahlenempfindlichkeit der HPV- positiven OSCC gibt, bestehen derzeit in der Praxis noch keine Unterschiede im Behandlungskonzept der OSCC in Abhängigkeit vom HPV-Status. Es ist jedoch davon auszugehen, dass in Zukunft für bestimmte OSCC- Patientengruppen mit niedrigem Risiko eine Therapiedeeskalation ermöglicht wird.}

\section{Korrespondenzadresse}

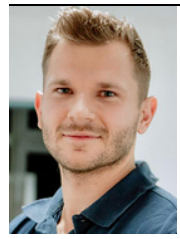

\section{A. Riders}

Klinik für Hals-Nasen-

Ohrenheilkunde, Universitätsklinikum Münster

Kardinal-von-Galen-Ring 10, 48149 Münster, Deutschland Armands.Riders@ ukmuenster.de armands.riders@gmail.com

Funding. Open Access funding enabled and organized by Projekt DEAL.

\section{Einhaltung ethischer Richtlinien}

Interessenkonflikt. A. Riders, M. Oberste, B. Abbaspour, A. Beule und C. Rudack geben an, dass kein Interessenkonflikt besteht.

Alle beschriebenen Untersuchungen am humanen Gewebe wurden mit Zustimmung der zuständigen Ethik-Kommission durchgeführt.

Open Access. Dieser Artikel wird unter der Creative Commons Namensnennung 4.0 International Lizenz veröffentlicht, welche die Nutzung, Vervielfältigung, Bearbeitung, Verbreitung und Wiedergabe in jeglichem Medium und Format erlaubt, sofern Sie den/die ursprünglichen Autor(en) und die Quelle ordnungsgemäß nennen, einen Link zur Creative Commons Lizenz beifügen und angeben, ob Änderungen vorgenommen wurden.

Die in diesem Artikel enthaltenen Bilder und sonstiges Drittmaterial unterliegen ebenfalls der genannten Creative Commons Lizenz, sofern sich aus der Abbildungslegende nichts anderes ergibt. Sofern das betreffende Material nicht unter der genannten Creative Commons Lizenz steht und die betreffende Handlung nicht nach gesetzlichen Vorschriften erlaubt ist, ist für die oben aufgeführten Weiterverwendungen des Materials die Einwilligung des jeweiligen Rechteinhabers einzuholen.

Weitere Details zur Lizenz entnehmen Sie bitte der Lizenzinformation auf http://creativecommons.org/ licenses/by/4.0/deed.de.

\section{Literatur}

1. Baumeister P, Rauch J, Jacobi C et al (2017) Impact of comorbidity and anemia in patients with oropharyngeal cancer primarily treated with surgery in the human papillomavirus era. Head Neck39(1):7-16

2. ChaturvediAK, EngelsEA, Anderson WF, Gillison ML (2008) Incidence trends for human papillomavirusrelated and -unrelated oral squamous cell carcinomas in the United States. J Clin Oncol 26(4):612-619

3. Chaturvedi AK, Engels EA, Pfeiffer RM et al (2011) Human papillomavirus and rising oropharyngeal cancer incidence in the United States. J Clin Onco 29(32):4294-4301

4. D'Souza G, Kreimer AR, Viscidi R et al (2007) Case-control study of human papillomavirus and oropharyngeal cancer. $\mathrm{N}$ Engl J Med 356(19):1944-1956

5. Dahlstrom KR, Bell D, Hanby D et al (2015) Socioeconomic characteristics of patients with oropharyngeal carcinoma according to tumor HPV status, patient smoking status, and sexual behavior. Oral Oncol 51(9):832-838

6. Deschuymer S, Dok R, Laenen A, Hauben E, Nuyts S (2018) Patient selection in human papillomavirus related oropharyngeal cancer: the added value of prognostic models in the new TNM 8th edition era. Front Oncol 8:273

7. Fazel A, Quabius ES, Fabian A et al (2020) The influence of smoking and co-morbidity on dose achievement in primary or adjuvant radio(chemo)therapy in head and neck squamous cell carcinoma (HNSCC). Front Oncol 10:398

8. Fazel A, Quabius ES, Gonzales-Donate $M$ et al (2020) Alteration of smoking habit at time of first diagnosis influences survival of patients with HNSCC. Mol Clin Oncol 13(5):50

9. Gillison ML, Trotti AM, Harris J et al (2019) Radiotherapy plus cetuximab or cisplatin in human papillomavirus-positive oropharyngeal cancer (NRG Oncology RTOG 1016): a randomised, multicentre, non-inferiority trial [published correction appears in Lancet. 2020, 395(10226):784. Lancet 393(10166):40-50

10. Grønhøj C, Jensen JS, Wagner S et al (2019) Impact on survival of tobacco smoking for cases with oropharyngeal squamous cell carcinoma and known human papillomavirus and p16-status: a multicenter retrospective study. Oncotarget 10(45):4655-4663

11. Grønhøj C, Kronberg Jakobsen K, Kjær E, Friborg J von Buchwald C (2019) Comorbidity in HPV+ and HPV-oropharyngeal cancer patients: a populationbased, case-control study. Oral Oncol 96:1-6

12. Javadi P, Sharma A, Zahnd WE, Jenkins WD (2017) Evolving disparities in the epidemiology of oral cavity and oropharyngeal cancers. Cancer Causes Control 28(6):635-645

13. Kimple RJ, Smith MA, Blitzer GC et al (2013) Enhanced radiation sensitivity in HPV-positive head and neck cancer. Cancer Res 73(15):4791-4800

14. Mahal BA, Catalano PJ, Haddad RI et al (2019) Incidence and demographic burden of HPVassociated oropharyngeal head and neck cancers in the United States. Cancer Epidemiol Biomarkers Prev 28(10):1660-1667

15. Masterson L, Moualed D, Liu ZW et al (2014) Deescalation treatment protocols for human papillomavirus-associated oropharyngeal squamous cell carcinoma: a systematic review and metaanalysis of current clinical trials. Eur J Cancer 50(15):2636-2648
16. Mehanna H, Robinson M, Hartley A et al (2019) Radiotherapy plus cisplatin or cetuximab in low risk human papillomavirus-positive oropharyngeal cancer (De-ESCALaTE HPV): an open-label randomised controlled phase 3 trial. Lancet 393(10166):51-60

17. Modesto A, Galissier T, Lusque A et al (2019) Definitive Radiochemotherapie oder initiale Operation beim Oropharynxkarzinom: In welchem Umfang kann die p16-Expression im Entscheidungsprozess verwendet werden? Strahlenther Onkol 195(6):496-503

18. Morbini P, Alberizzi P, Ferrario G et al (2019) The evolving landscape of human papillomavirusrelated oropharyngeal squamous cell carcinoma at a single institution in Northern Italy. Acta Otorhinolaryngol Ital 39(1):9-17

19. Nauta IH, Rietbergen MM, van Bokhoven AAJDetal (2018) Evaluation of the eighth TNM classification on p16-positive oropharyngeal squamous cell carcinomas in the Netherlands and the importance of additional HPV DNA testing. Ann Oncol 29(5):1273-1279

20. Quabius ES, Fazel A, Knieling C et al (2020) No association between HPV-status in tonsillar tissue and sexual behavior of the patients in a northern German population-critical view of the link between HPV natural history and HPV-driven carcinogenesis. Papillomavirus Res 10:100207

21. Rieckmann T, Tribius S, Grob TJ et al (2013) HNSCC cell lines positive for HPV and $p 16$ possess higher cellular radiosensitivity due to an impaired DSB repair capacity. Radiother Oncol 107(2):242-246

22. Stenmark MH, Shumway D, Guo C et al (2017) Influence of human papillomavirus on the clinical presentation of oropharyngeal carcinoma in the United States. Laryngoscope 127(10):2270-2278

23. Wagner S, Prigge ES, Wuerdemann $\mathrm{N}$ et al (2020) Evaluation of p16INK4a expression as a single marker to select patients with HPVdriven oropharyngeal cancers for treatment deescalation. Br J Cancer 123(7):1114-1122

24. Wittekindt C, Wagner S, Bushnak A et al (2019) Increasing incidence rates of oropharyngeal squamous cell carcinoma in Germany and significance of disease burden attributed to human papillomavirus. Cancer Prev Res 12(6):375-382

25. Wittekindt C, Wagner S, Sharma SJ et al (2018) HPV - a different view on head and neck cancer. Laryngorhinootologie 97(S01):S48-S113

26. Wuerdemann N, Wittekindt C, Sharma SJ et al (2017) Risk factors for overall survival outcome in surgically treated human papillomavirus-negative and positive patients with oropharyngeal cancer. Oncol Res Treat 40(6):320-327

27. Würdemann N, Wagner S, Sharma SJ et al (2017) Prognostic impact of AJCC/UICC 8th edition: new staging rules in oropharyngeal squamous cell carcinoma. Front Oncol 7:129 